\section{PP IMPROVING HEALTHCARE SERVICE DELIVERED IN MULTIPLE MYELOMA CLINIC - MEDICINE MEETS HEALTH PSYCHOLOGY TO EXPLORE PATIENTS' VIEWS ON A PROPOSAL TO CHANGE ROUTINE FOLLOW-UP}

M Heinrich, ${ }^{1} \mathrm{D}$ De-Silva, ${ }^{2} \mathrm{~J}$ Land, ${ }^{1 *} \mathrm{~K}$ Yong ${ }^{2} .{ }^{1}$ Department of Behavioural Science and Health, University College London, UK; ${ }^{2}$ Cancer Institute, University College London, UK

\subsection{6/bmjopen-2017-016492.29}

Background: Qualitative research (QR) has permeated into the areas of 'hard science', including medicine. Still, there remains criticism about the suitability of QR for the medical context. In contrast, the QR experts argue that high level of skill in conducting qualitative interviews and analysis is required as the lack of such may lead to poor data collection or invalid conclusions. Despite these reservations clinicians (UCLH) and academics (UCL) designed a qualitative study which will inform the decisionmaking process related to the set-up of a telephone clinic for patients with myeloma through an exploration of the benefits and barriers to using such service. This presentation is a reflection on the collaboration between academics and clinicians.

Learnings: Qualitative interviewing differs from the instructive style of communication doctors use, but the collaborating clinicians were open to learn it and felt that it allowed them to become less paternalistic, and turn towards a holistic approach to negotiating health services with patients. The use of QR was viewed as an opportunity for a doctor-patient dialog about the development of a service that is safe and acceptable both to patients and clinicians, time-efficient and empowering patients to manage their illness.

Conclusion: QR provided added value to the work of medical professionals and was used to identify patients' needs in the context of a proposed healthcare system. The interviews created an opportunity to ensure that patients' opinions were heard and used to inform the development of a service in a way that is acceptable to the patients. 